\title{
Identification and Quantification of Heterogeneously- methylated DNA Fragments Using Epiallele-sensitive Droplet Digital Polymerase Chain Reaction (EAST-ddPCR)
}

\author{
MARIO MENSCHIKOWSKI ${ }^{1}$, CARSTEN JANDECK $^{1}$, MARKUS FRIEDEMANN ${ }^{1}$, \\ SUSAN RICHTER ${ }^{1}$, DANA THIEM ${ }^{2}$, BJÖRN SÖNKE LANGE ${ }^{2}$ and MEINOLF SUTTORP ${ }^{2}$ \\ ${ }^{1}$ Institute of Clinical Chemistry and Laboratory Medicine, ${ }^{2}$ Department of Pediatrics, \\ University Hospital "Carl Gustav Carus", Technical University of Dresden, Dresden, Germany
}

\begin{abstract}
Background/Aim: DNA methylation plays an important role in the initiation and propagation of carcinogenesis; however, the role of heterogeneously methylated epialleles is currently not well studied, also due to the lack of sensitive, unbiased and high throughput methods. Here, a newly developed droplet digital PCR (ddPCR)-based method was evaluated regarding its ability to quantify such heterogeneously methylated epialleles with sufficient analytical sensitivity and specificity. Materials and Methods: Genomic DNA from blood leukocytes and bone marrow aspirate of an 8-year old male with $B$-cell acute lymphoblastic leukemia $(B-A L L)$ and from normal and malignant prostate cell lines were analysed using ddPCR. Results: By using these DNA samples, the specificity of an applied set of fluorescence-labeled probes was demonstrated as a proof of concept. Conclusion: All individual heterogeneouslymethylated epialleles were quantifiable by a set of fluorescencelabeled probes with complementary sequences to epialleles in a closed-tube and high-throughput manner. The new method named epiallele-sensitive droplet digital PCR (EAST-ddPCR) may give new insights in the generation and regulation of epialleles and may help in finding new biomarkers for the diagnosis of benign und malignant diseases.
\end{abstract}

Epigenetic mechanisms such as DNA methylation play an important role in many physiological and pathophysiological

This article is freely accessible online.

Correspondence to: Mario Menschikowski, Institute of Clinical Chemistry and Laboratory Medicine, Medical Faculty Carl Gustav Carus, Fetscherstrasse 74, D 01307 Dresden, Germany. Tel: +49 3514582634, Fax: +49 3514584332, e-mail: Mario.Menschikowski@uniklinikum-dresden.de

Key Words: Digital PCR, DNA methylation, heterogeneously methylated epialleles, leukemia, prostate cancer cells. processes including cancerogenesis (1-8). As a consequence of DNA methylation, genes coding for tumor suppressors can be silenced leading to attenuation of anti-cancerous processes, such apoptosis and senescence, and hence constant proliferation. Based on the observation that changes in methylation pattern occur early in carcinogenesis analyses of aberrant DNA methylation patterns have attracted considerable interest as potential new biomarkers for the early detection of cancer onset as well as disease monitoring after treatment (1-8).

In the past, methylation of target genes was assessed with PCR-based approaches using bisulfite treated DNA. The popular method of methylation-specific (MS)-PCR is, however, not able to detect heterogeneously methylated epialleles (9). In contrast, the more recently developed method of methylation-sensitive high-resolution melt (MSHRM) analysis generates more complex melt curves indicating the presence of heterogeneously methylated epialleles, but lacks the possibility of detailed analysis and quantification (9). Due to growing recognition of the potential importance of heterogeneously methylated epialleles for the identification of new biomarkers in early cancer diagnosis, several new techniques have been developed, which are termed digital methylation sensitiveHRM or digital MethyLight $(10,11)$. A disadvantage of the latter is that currently available PCR-based measuring instruments allow HRM analyses only in a restricted number of reaction compartments, for example in 96- or 384reaction-well-plate formats. Therefore, the analytical sensitivity and precision of this technique is limited. Other techniques, such as next generation sequencing, pyrosequencing and SequenomMassArray, are able to quantify heterogeneously methylated epialleles, but high upfront investment and consumable costs hinder their establishment in routine analytical laboratories $(9,12-14)$.

The current study describes a new method based on digital PCR that uses distinct fluorescence-labeled probes allowing 


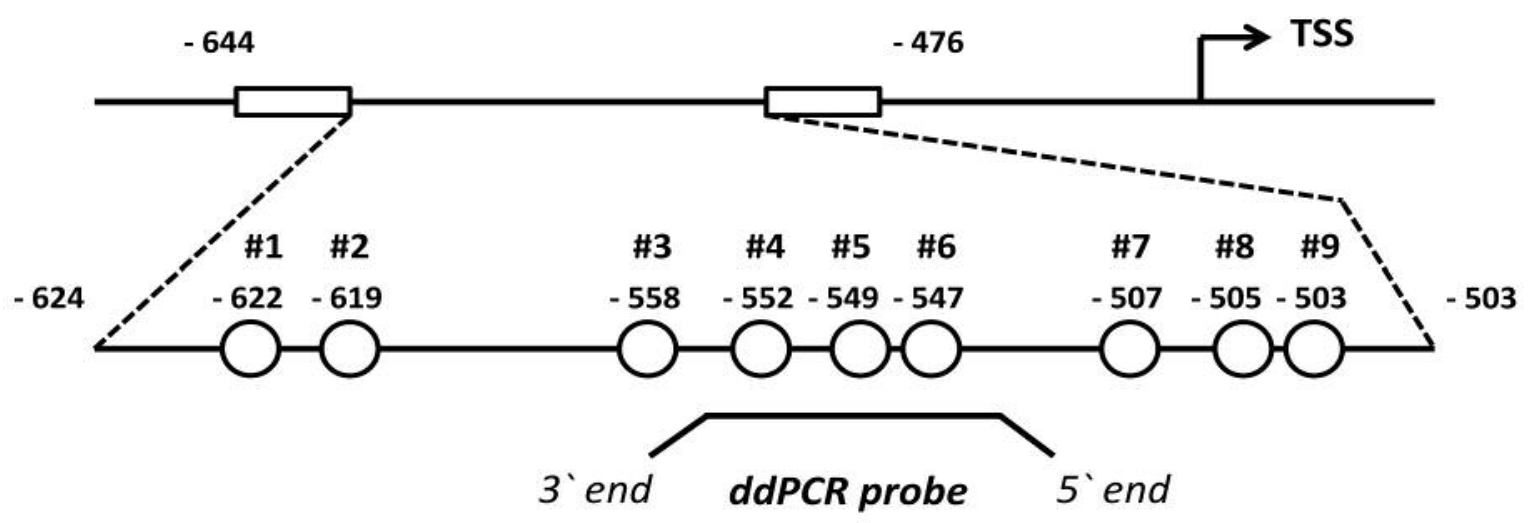

Figure 1. Schematic illustration of primer localization and fluorescence-labeled probes in dependence on the presence of CpG sites analyzed in the study and applied in MS-HRM and ddPCR analyses. Methylation-independent primers amplified an amplicon with the length of 168 bp from -644 bp to -476 bp upstream of the transcription start site (TSS). MS-HRM analysis covered 9 CpG sites (\#1 to \#9). For ddPCR eight different probes with sequences complementary to heterogeneously methylated and unmethylated epialleles were used. These eight different epialleles were identified and quantified based on methylation of CpG-sites \#4 to \#6 (position 4: -547 bp, position 5: -549 bp and position 6: -552 bp).

the identification and quantification of $3 \mathrm{CpG}$-sites associated with heterogeneously methylated M-type phospholipase A2 receptor $(P L A 2 R 1)$ epialleles. As a proof of concept, genomic DNA from two samples of a B-ALL patient and from different prostate cell lines, for which the presence of heterogeneously methylated epialleles was initially identified using MS-HRM technique, were examined. PLA2R1 was shown to play a crucial role in several anti-tumour and anti-inflammatory responses including apoptosis, replicative- and stress-induced senescence, and inhibition of cell transformation (7, 15-18). The discovery of $P L A 2 R 1$ promoter hypermethylation connected with lowered PLA2R1 expression in leukemic cells indicated a tumour-suppressive role of this receptor (19). The observed $P L A 2 R 1$ promoter methylation was not restricted to leukemia, but also observed in breast cancer cell lines (20) and in clear cell renal cell carcinomas (21) and is also present in prostate cancer cell lines as shown in this study.

\section{Materials and Methods}

Patient samples. Peripheral EDTA-blood and bone marrow aspirate were collected from an 8-year old male patient with a relapse of BALL and morphologically detected $4 \%$ blood and $61 \%$ bone marrow blasts. Parents of the patient provided written informed consent for the following studies. Use of the patient's samples was approved by the Ethical Board of the University Hospital of Dresden.

Cell culture. Normal human prostate epithelial cells, PrEC, were purchased from Cambrex Bio Science (Walkersville, MD, USA) and maintained up to a maximum of five passages in prostate epithelial growth medium supplemented with bovine pituitary extract, epidermal growth factor, insulin, transferrin, hydrocortisone, retinoic acid, epinephrine, triiodothyronine, and gentamicin-amphotericin solution on dishes coated with collagen type I (BioCoat; BD Falcon,
Heidelberg, Germany). Every 2 to 3 days, the medium was changed, and before reaching confluence, the cells were passaged using trypsin/ethylenediaminetetraacetic acid. Human prostatic malignant prostate cell lines, LNCaP, PC-3, and DU-145 were attained from the German Collection of Microorganisms and Cell Cultures (DSMZ, Braunschweig, Germany) and cultured in RPMI 1640 (Invitrogen, Darmstadt, Germany) supplemented with 10\% heatinactivated fetal calf serum (FCS, Lonza, Köln, Germany), $2 \mathrm{mM}$ L-glutamine, $100 \mathrm{U} / \mathrm{ml}$ penicillin, and $100 \mu \mathrm{g} / \mathrm{ml}$ streptomycin at $37^{\circ} \mathrm{C}$ in a humidified atmosphere of $5 \% \mathrm{CO}_{2}$. Benign prostatic hyperplastic epithelial cell line, BPH-1 was purchased from DSMZ and cultured in RPMI-1640 medium supplemented with $20 \%$ FCS, $20 \mathrm{ng} / \mathrm{ml}$ testosterone, $5 \mu \mathrm{g} / \mathrm{ml}$ transferrin, $5 \mathrm{ng} / \mathrm{ml}$ sodium selenite, $5 \mu \mathrm{g} / \mathrm{ml}$ insulin and antibiotics.

Extraction of genomic DNA and bisulfite modification. Genomic DNA was isolated from peripheral blood, bone marrow aspirate, and prostatic cell lines using a Blood \& Cell Culture DNA Mini Kit (Qiagen GmbH, Hilden, Germany) following the manufacturer's instructions. Aliquots of $500 \mathrm{ng}$ of isolated genomic DNA were bisulfite modified using the EpiTect Bisulfite Kit (Qiagen $\mathrm{GmbH}$ ) according to manufacturer's instructions. Levels of ssDNA after bisulfite modification were determined using the Quantifluor ssDNA-System (Promega GmbH, Mannheim, Germany).

Methylation-specific high-resolution melt (MS-HRM) analyses. MSHRM analyses were carried out to quantify the extent of methylation in the distinct region -644 bp to -478 bp from the transcription start site (TSS) of the PLA2R1 gene (Figure 1 and [19]). These analyses were carried out using Rotor-Gene Q (Qiagen $\mathrm{GmbH}$ ) and the EpiTect MS-HRM PCR Kit according to manufacturer's instructions. $2 \mu \mathrm{l}$ of bisulfite modified genomic DNA (containing 4-30 ng ssDNA) were applied and $10 \mathrm{ng}$ bisulfite modified unmethylated $(0 \%)$ and methylated (100\%) standard DNA (Qiagen $\mathrm{GmbH}$ ) were used as positive controls and non-template and $30 \mathrm{ng}$ genomic DNA without bisulfite modifications as negative controls in each run. PCR was performed in $12.5 \mu \mathrm{l}$ volumes. The applied methylation-independent 
Table I. Characteristics of single-strand DNA probes applied in the ddPCR. Closed circles indicate methylated, open circles unmethylated CpGsites (position 4: -547 bp, position 5: -549 bp and position 6: -552 bp upstream from the transcription start site as shown in Figure 1).

\begin{tabular}{|c|c|c|c|}
\hline ID & fluorescence/quencer & sequences $\left(5^{\prime} \rightarrow 3^{\prime}\right)$ & schematic illustration $\left(5^{\prime} \rightarrow 3^{\prime}\right)$ \\
\hline PLA2R1-1a & 5'-FAM, 3'-BHQ & CCCAACTACTCCACAACGCAA & $\stackrel{6}{0}$ \\
\hline PLA2R1-1b & 5'-FAM, 3'-BHQ & CCCAACTACTCCACGACACAA & $\begin{array}{c}6 \\
0\end{array}$ \\
\hline PLA2R1-1C & 5'-FAM, 3'-BHQ & CCCAACTACTCCGCAACACAA & \\
\hline PLA2R1-2a & 5'-FAM, 3'-BHQ & CCCAACTACTCCACGACGCAA & \\
\hline PLA2R1-2b & 5'-FAM, 3'-BHQ & CCCAACTACTCCGCGACACAA & \\
\hline PLA2R1-2c & 5'-FAM, 3'-BHQ & CCCAACTACTCCGCAACGCAA & \\
\hline PLA2R1-3 & 5'-FAM, 3'-BHQ & CCCAACTACTCCGCGACGCAA & \\
\hline PLA2R1-0 & 5'-HEX, 3'-BHQ & CCCAACTACTCCACAACACAA & $\begin{array}{ccc}6 & 5 & 4 \\
-0 & 0 & 0\end{array}$ \\
\hline
\end{tabular}

primer (MIP) pairs were 5'-GGG GTA AGG AAG GTG GAG AT3' and 5'-ACA AAC CAC CTA AAT TCT AAT AAA CAC-3', generating PCR products of a length of $168 \mathrm{bp}$. The primers were applied at a final concentration of $0.8 \mu \mathrm{M}$. The conditions of amplification were as follows: 40 courses at $95^{\circ} \mathrm{C}$ for 10 seconds, $58^{\circ} \mathrm{C}$ for $30 \mathrm{sec}$ and $72^{\circ} \mathrm{C}$ for $15 \mathrm{sec}$. Immediately after PCR, products were analyzed by high resolution melt analysis with fluorescence measured during the linear temperature transition from $50-95^{\circ} \mathrm{C}$ at $0.01^{\circ} \mathrm{C} / \mathrm{sec}$.

Digital droplet PCR of bisulfite-modified DNA. All ddPCR analyses were performed using the QX100 Droplet Digital PCR System according to the manufacturer's instructions (Bio-Rad $\mathrm{GmbH}$, München, Germany). Each ddPCR reaction mixture consisted of the $2 \mathrm{X}$ ddPCR Supermix for primers and probes. Primer and probe sequences were designed using Oligo Architec software from Sigma-Aldrich Chemicals GmbH (Taufkirchen, Germany) and are shown in Figure 1 and Table I. Probes were synthesized at 5'-end with FAM for heterogeneously methylated epialleles and with HEX for the quantification of unmethylated DNA fragments and at the 3 '-end with BHQ-1 as fluorescence quencher, respectively, and all ddPCR were performed as duplex assay containing simultaneously FAM- and the HEX-labelled probes.

MIP as used in MS-HRM and probes were used at final concentrations of $900 \mathrm{nmol} / \mathrm{l}$ and $250 \mathrm{nmol} / 1$, respectively. Each $20 \mu \mathrm{l}$ PCR reaction containing $2 \mu \mathrm{l}$ of bisulfite modified genomic DNA or negative controls were loaded into the Bio-Rad DG8 disposable droplet generation cartridges (Bio-Rad). A volume of $70 \mu \mathrm{l}$ of droplet generation oil was loaded into adjacent wells. Then, microfluidic chips were loaded into a droplet generator (Bio-Rad). In general, 10,000 to 17,000 accepted droplets per reaction were generated. The water-inoil droplets were pipette-transferred from the outlet well to a 96-well polypropylene plate. The heat-sealed plate was placed on a T100 Thermal Cycler (Bio-Rad) and amplified for 40 cycles to the endpoint. The thermal cycling conditions were $95^{\circ} \mathrm{C}$ for $10 \mathrm{~min}$ and 40 cycles of $94{ }^{\circ} \mathrm{C}$ for $30 \mathrm{~s}$ and $58.8{ }^{\circ} \mathrm{C}$ for $1 \mathrm{~min}$ with a final $10 \mathrm{~min}$ hold at 98 ${ }^{\circ} \mathrm{C}$. After PCR amplification, the 96-well plate was loaded into a QX100 droplet reader (Bio-Rad).

In preliminary investigations, the efficiency of fluorescence signal generation of FAM and HEX labelled probes for fully methylated and unmethylated epialleles was studied. The annealing temperature was chosen in such a way, that a maximum of separation of the FAM-positive subfractions with simultaneous sufficient HEX signal generation resulted. The same temperature was used in cases of six additional probes for heterogeneously methylated epialleles whose melt properties should be behave similar to those complementary to fully methylated and unmethylted DNA fragments.

All methylation quantification experiments included no-template controls (NTCs), which contained all the components of the reaction but without DNA templates. In addition, $30 \mathrm{ng}$ of genomic DNA (gDNA) without previous bisulfite modifications was analyzed as a negative control. Data were processed using the QuantaSoft software version 1.6.6.0320 (Bio-Rad). 

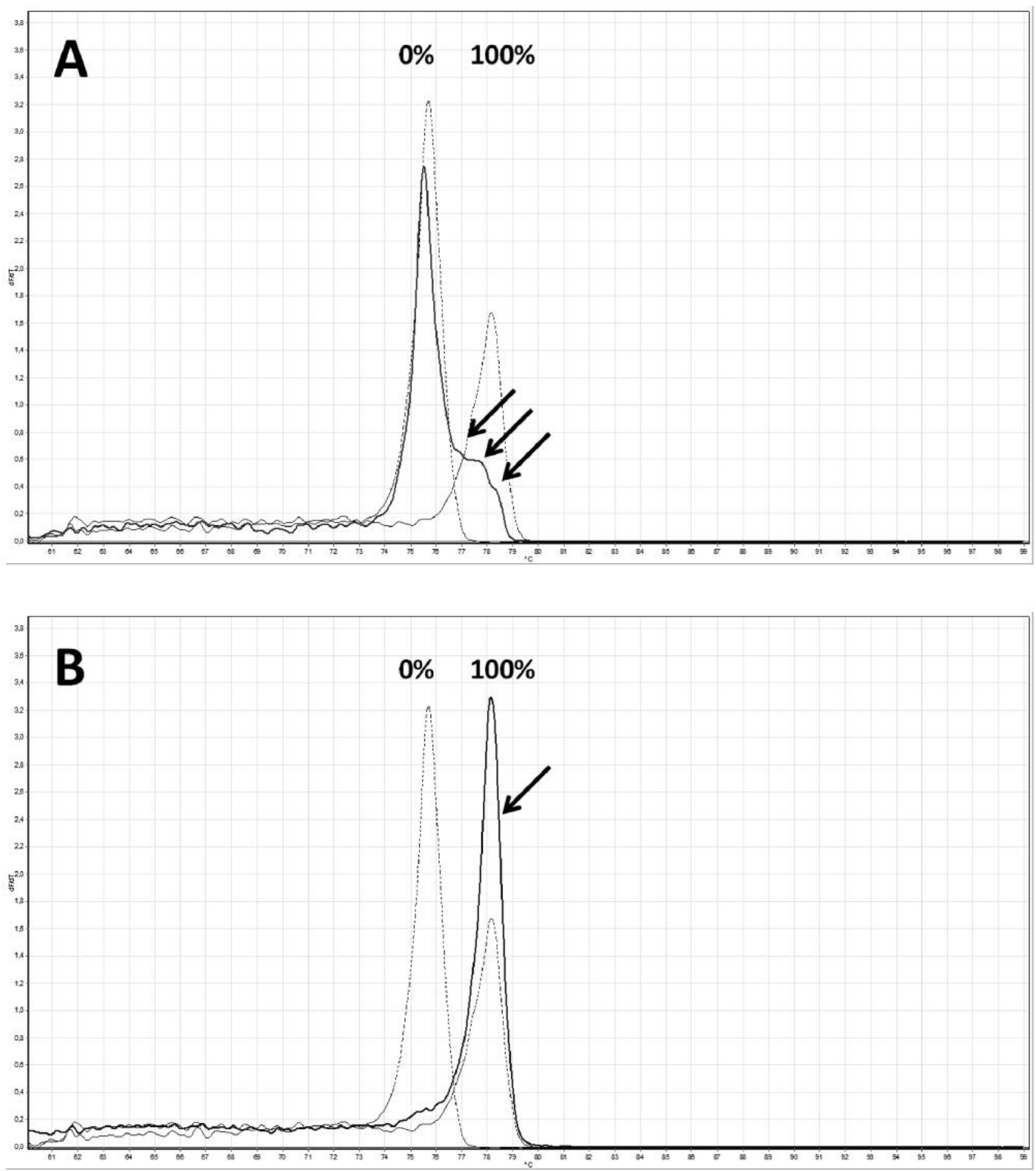

Figure 2. Methylation of the PLA2R1 gene in DNA samples isolated from blood leukocytes (A) and bone marrow aspirate (B) of an 8-year old male with relapsed B-ALL. MS-HRM analyses of amplified PLA2R1 sequences covering nine 5'-CpG-sites (as shown schematically in Figure 1) after isolation and subsequent bisulfite modification of genomic DNA. Melt profiles as negative first derivative of the raw melt pattern (-dF/dT) of 0\% and $100 \%$ methylated standard DNA samples (dotted lines) and DNA from blood leukocytes (A) and bone marrow aspirate (B) are shown. Arrows in (A) indicate three heterogeneously methylated subfractions in addition to the unmethylated fraction, whereas in (B) the arrow indicates the major methylated fraction in addition to a minor unmethylated subfraction in the melt curve range of 0\% and 100\% methylated DNA standard controls, respectively. Each sample was analyzed in duplicate by MS-HRM resulting in comparable melt profiles and are representative of two independent experiments. 
A

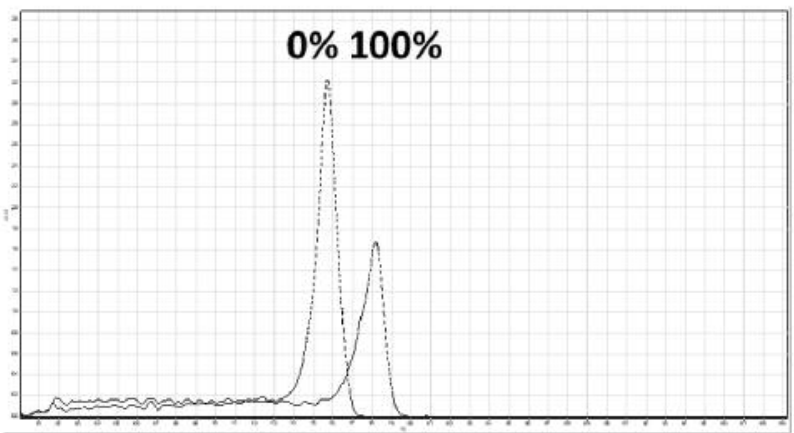

C

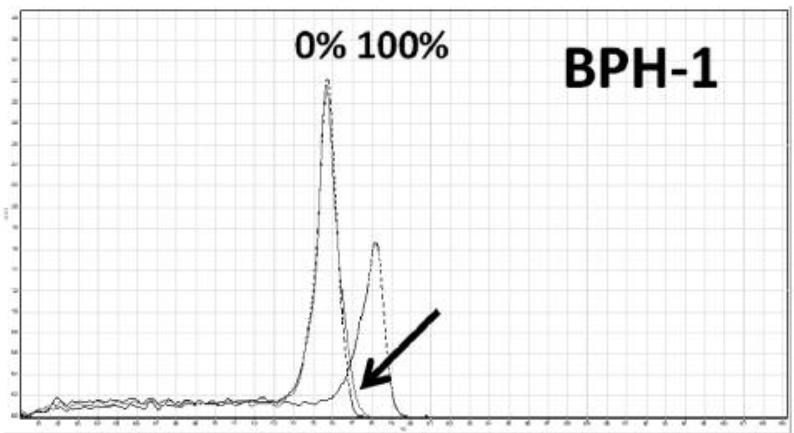

$\mathbf{E}$

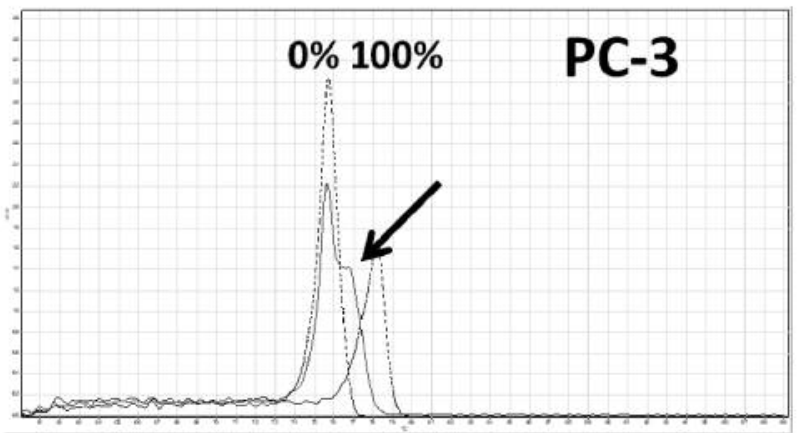

B

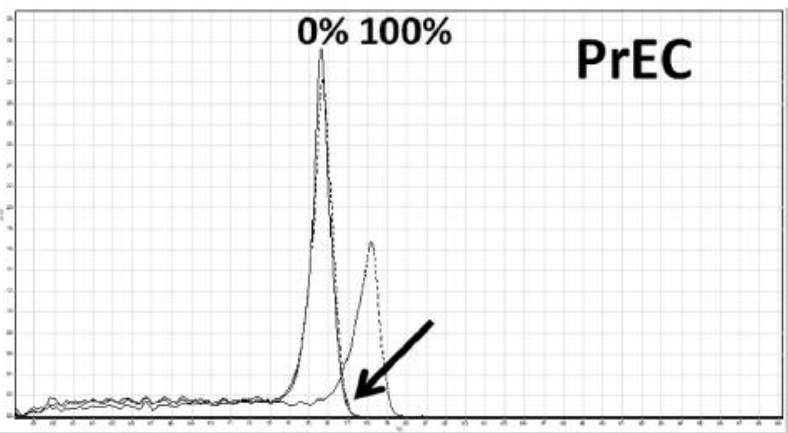

D

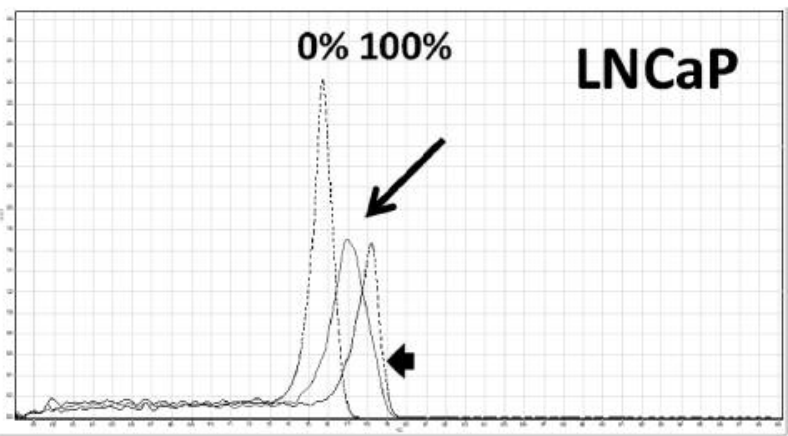

$\mathbf{F}$

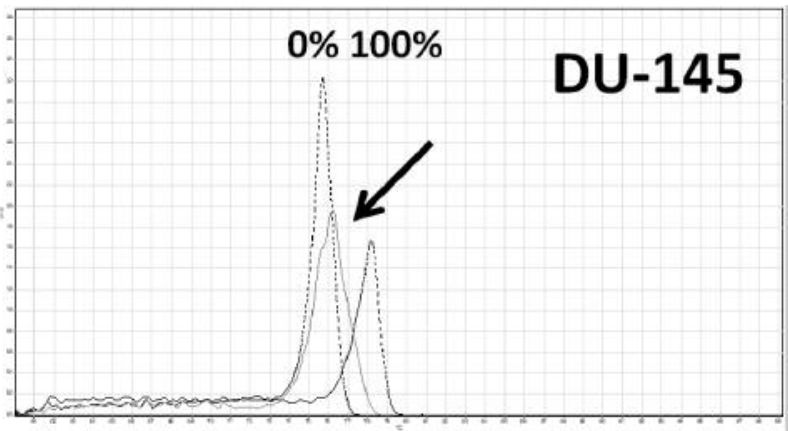

Figure 3. Methylation of the PLA2R1 gene in DNA samples isolated from normal prostate epithelial cells PrEC (B), benign prostate hyperplasic cell line BPH-1 (C), and malignant prostate cancer cell lines LNCaP $(D), P C-3(E)$, and DU-145 (F). MS-HRM analyses of amplified PLA2R1 sequences covering nine 5'-CpG-sites (as shown schematically in Figure 1) after isolation and subsequent bisulfite modification of genomic DNA. Melt profiles are displayed as negative first derivative of the raw melt pattern (-dF/dT) of $0 \%$ and $100 \%$ methylated standard DNA samples (dotted lines in $[A-F])$. Arrows $(B-F)$ indicate the melt profiles of the analyzed DNA samples isolated from the prostate cell lines. Each sample was analyzed in duplicate by MS-HRM resulting in comparable melt profiles and are representative of two independent experiments.

Ethics approval and consent to participate. The study was approved by the Ethics Committee of the University Hospital 'Carl Gustav Carus', Dresden, Germany, with the reference number AZ1055112 and written informed consent was obtained from parents of the patient.

\section{Results}

MS-HRM analyses of DNA from peripheral blood, bone marrow aspirate, and prostate cell lines. MS-HRM 


\section{E188}
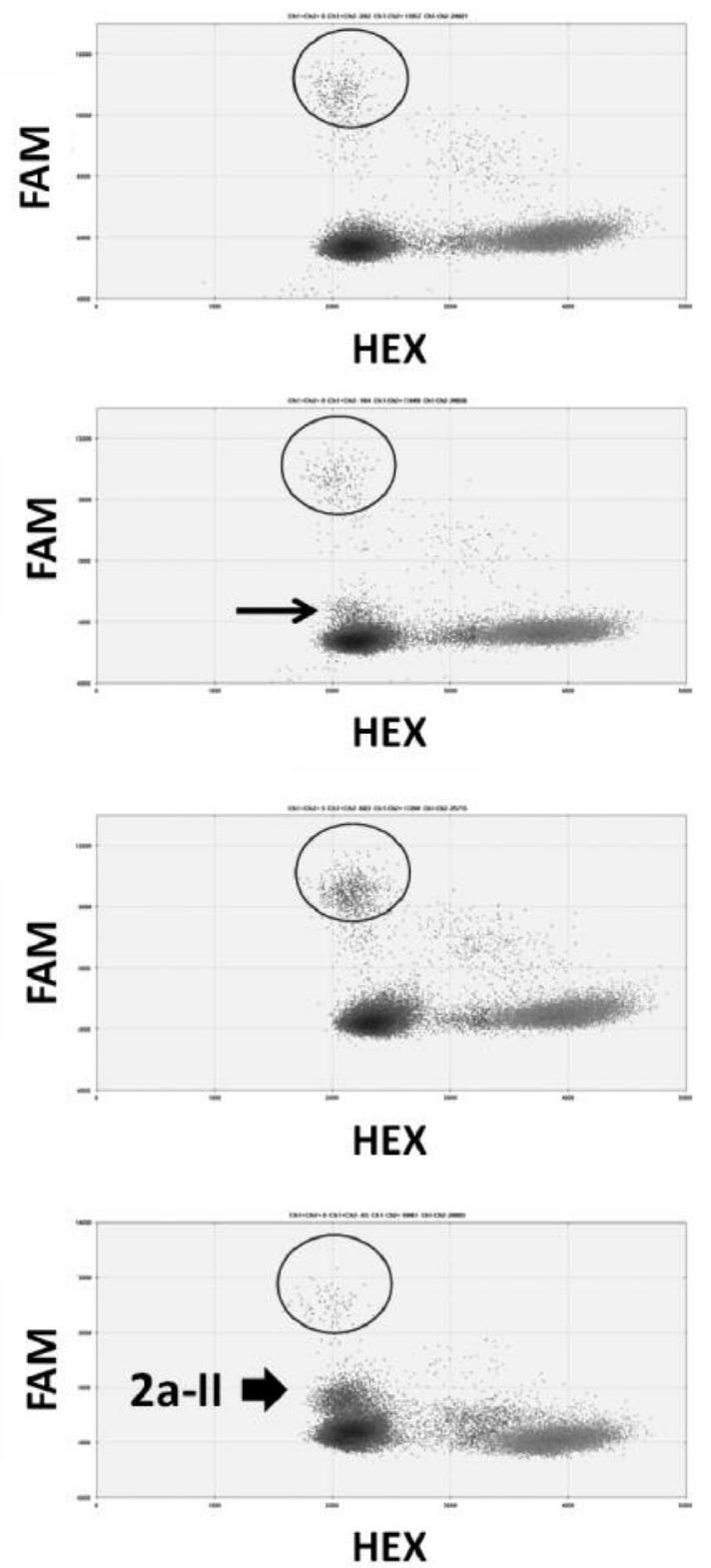

K189

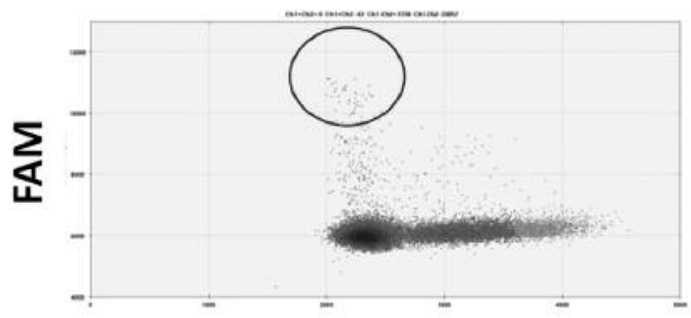

HEX
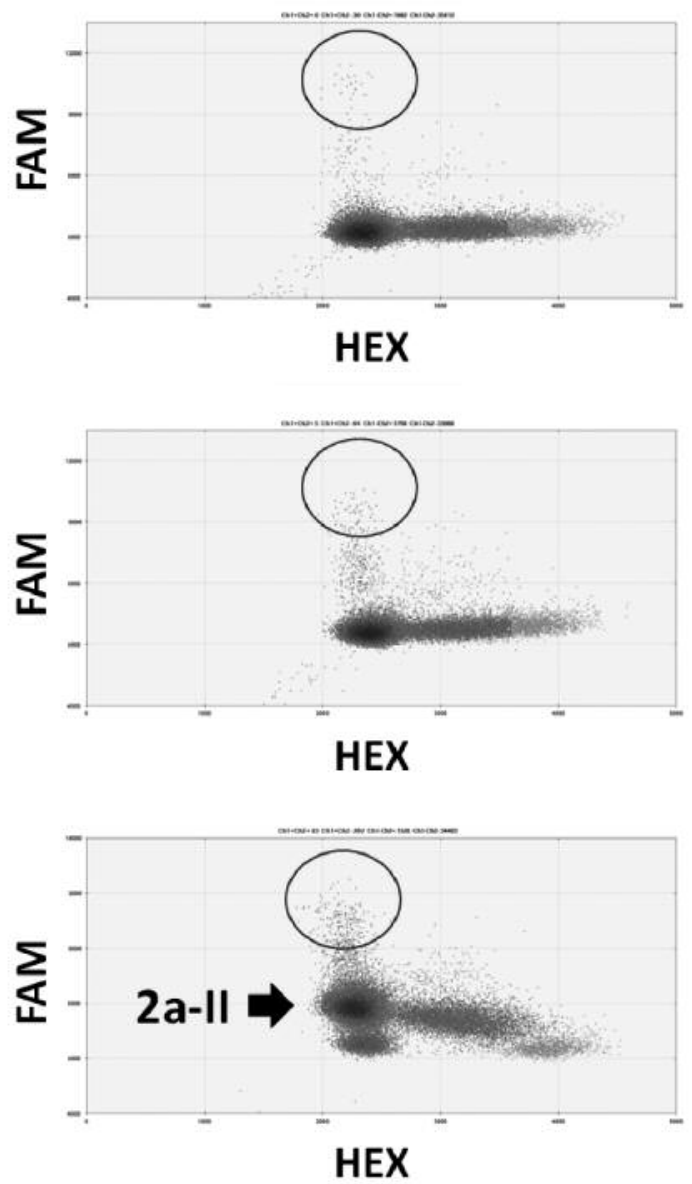

PLA2R1-1a

PLA2R1-1b

\section{PLA2R1-2a}

Figure 4. Continued

analyses demonstrated that patient leukocyte DNA (sample ID E188) exhibited at least three heterogeneously methylated epiallele subfractions and a major fraction of unmethylated DNA (Figure 2A). In contrast, bone marrow DNA from the same patient (sample ID K189) was almost fully methylated. The curve of the melt profile was nearly identical to those of the $100 \%$-methylated standard DNA showing only a minor unmethylated subfraction in bone marrow DNA (Figure 2B).
In PrEC cells, originating from normal prostate, the melt curve profile mimicked that of the unmethylated standard DNA (Figure 3A/B). In benign hyperplastic prostate cells, BPH-1, the melt profile was slightly shifted to the right, indicating a low amount of methylated DNA (Figure 3C). LNCaP cells, a malignant prostate cancer line, had a strong shift to the methylated side; however, with exception of a small part (marked by arrow head in Figure 3D), the peak did not overlay with the $100 \%$-methylated standard (Figure 3D). In PC-3 cells, 
E188

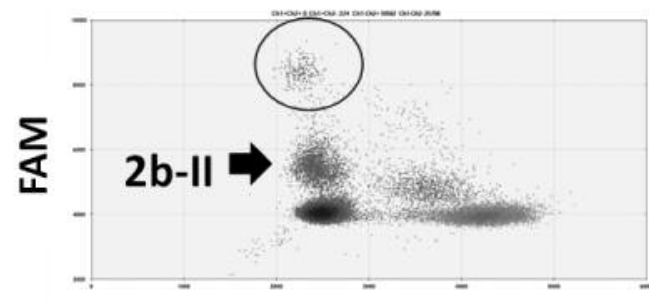

HEX

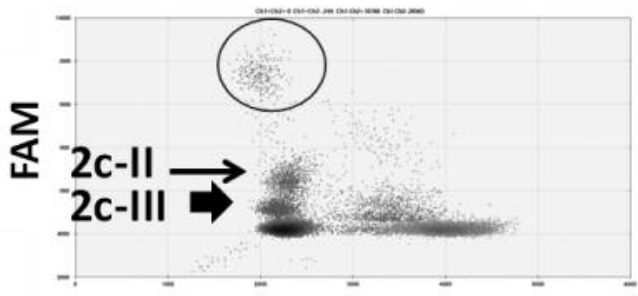

HEX
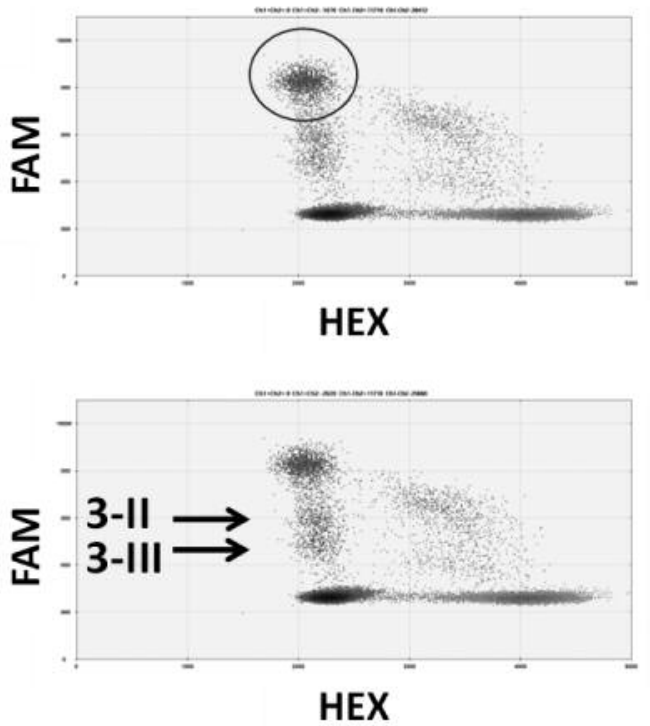

K189

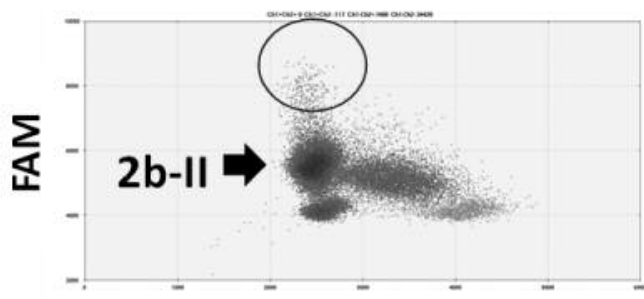

HEX

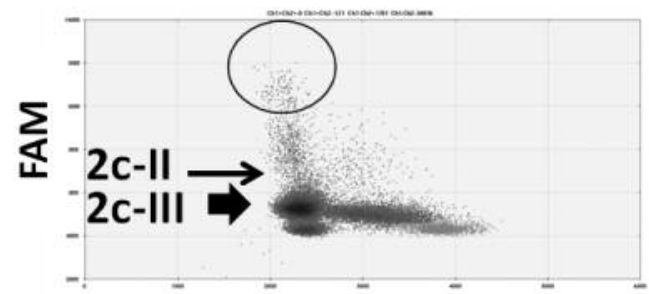

HEX
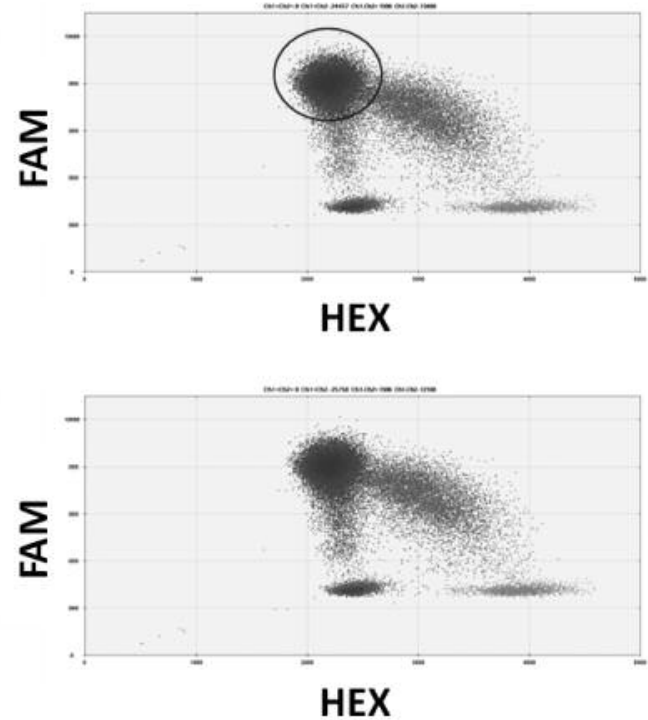

PLA2R1-2b

PLA2R1-2c

PLA2R1-3s

PLA2R1-3w

Figure 4. 2D-projection of FAM-signals (positive for methylated DNA fragments) and HEX-signals (positive for unmethylated DNA fragments) in DNA samples isolated from blood leukocytes (on the left side, E188) and bone-marrow aspirate (on the right side, K189) after 40 cycles of ddPCR. FAM signals marked by circles were used for calculation. On the right side, probe identifications, PLA2R1-1a to -3 (refer to Table I) are shown. In the left graphic labeled with PLA2R1-1b the arrow marks a small cluster above the FAM signal of empty droplets. Cluster 2a-II, 2b-II and 2c-III corresponds to fully methylated epialleles. Clusters 3-II and 3-III were included in the quantification of PLA2R1-3-positive copies in PLA2R1-3w. In PLA2R1-3s the clusters 3-II and 3-III were excluded from quantification. DNA from blood leukocytes and bone-marrow aspirate were analyzed in triplicate and results are representative of two independent experiments.

most of the DNA was unmethylated with a smaller fraction being shifted to the methylated side, but not identical to the 100\%-methylated standard (Figure 3E). Similar results were obtained for DU-145 cells, where the majority of DNA was methylated and a smaller part unmethylated (Figure 3F).
ddPCR analyses of DNA from peripheral blood and bone marrow aspirate. Analysis by ddPCR was in agreement with MS-HRM results in that most of the bone marrow DNA from the B-ALL patient was fully methylated $(84.8 \%$, probe ID PLA2R1-3) with $13.6 \%$ being unmethylated (probe ID 
PLA2R1-0) and 1.7\% heterogeneously methylated (Figure 4, right panel with probe IDs PLA2R1-1a/1b/1c and PLA2R1$2 \mathrm{a} / 2 \mathrm{~b} / 2 \mathrm{c}$ and Table II).

In comparison, DNA from blood leukocytes of the same patient was only $14.2 \%$ fully methylated and $73.6 \%$ unmethylated (Figure 4, left panel and Table II). Probe PLA2R1-3 revealed the presence of subfractions with lower signal strength compared to the main subfraction with high signal strength found both in leukocyte and bone marrow DNA (Figure 4).

Using probes PLA2R1-2a/2b/2c several FAM-positive subfractions with varying signal strength were visible. Especially DNA from blood leucocytes (E188) showed a very distinct subfraction with strong signal amplitude for each of the probes (circles in Figure 4, left panel labeled with PLA2R1-2a/2b/2c). These DNA sub-fractions were clearly distinguishable from other populations with lower FAM signal amplitudes, and thresholds were set as shown by circles (Figure 4). The latter were applied to the bone marrow DNA (K189), where the fraction of epialleles was much lower compared to E188. Additionally, both samples presented with a large population characterized by a weak signal amplitude close to that of empty droplets (arrow heads in Figure 4, left and right panels labeled with PLA2R1$2 a / 2 b / 2 c)$. This fraction corresponds in size to that found in K189 and E188 using probe PLA2R1-3 and indicating fully methylated DNA (Figure 4 labeled with PLA2R1-3). PLA2R1-2c amplification of E188 produced another subfraction located between the probe-specific FAM fraction and the one corresponding to the PLA2R1-3 fraction (arrow in Figure 4, left panel labeled with PLA2R1-2c).

The dominant subfraction in E188 and K189 corresponding to fully methylated DNA was congruent with empty droplets using probes PLA2R1-1a/1b/1c (Figure 4, left and right panels labeled with PLA2R1-1a/1b/1c). These probes did not give a clear separation between the unmethylated subfraction and empty droplets. Instead it was necessary to use the number of unmethylated DNA copies measured with probes PLA2R1-2a/2b/2c/3. Despite insufficient separation of the unmethylated subfraction, probes PLA2R1-1a/1b/1c resulted in a confined fraction with high FAM intensity in E188 (circles in Figure 4). Probe PLA2R1-1b additionally produced a small subfraction slightly above the FAM signal of empty droplets but significantly separated from the cluster of specific FAM signals (arrow in Figure 4, left panel labeled with PLA2R1-1b). Due to almost fully methylated epialleles in $\mathrm{K} 189$ only minor fractions were detected with probes PLA2R1-1a/1b/1c, nicely reproducing the results from the MS-HRM analysis.

ddPCR analyses of DNA from normal and malignant prostate cancer cell lines. As a further proof of concept for the utility of the introduced ddPCR probes as identifiers for
Table II. Percentage amounts shown as mean $\pm S D$ of epiallele copies detected in DNA samples from blood leukocytes (E188) and bone marrow aspirate (K189) of an 8-years old male with relapsed B-ALL. After bisulfite modification genomic DNA samples were analyzed using ddPCR with eight different fluorescence-labeled probes (refer to Table I). Analyses were performed in triplicates and results are representative of two independent experiments.

\begin{tabular}{lrr}
\hline Probe ID & E188 & \multicolumn{1}{c}{ K189 } \\
\hline PLA2R1-1a & $1.8 \pm 0.3$ & $0.1 \pm 0.0$ \\
PLA2R1-1b & $1.0 \pm 0.1$ & $0.1 \pm 0.0$ \\
PLA2R1-1c & $4.2 \pm 0.3$ & $0.2 \pm 0.0$ \\
PLA2R1-2a & $0.5 \pm 0.2$ & $0.7 \pm 0.1$ \\
PLA2R1-2b & $1.4 \pm 0.3$ & $0.3 \pm 0.1$ \\
PLA2R1-2c & $1.5 \pm 0.1$ & $0.3 \pm 0.0$ \\
PLA2R1-3 & $10.9 \pm 0.5$ & $93.7 \pm 4.1$ \\
PLA2R1-0 & $78.8 \pm 0.7$ & $4.6 \pm 0.5$ \\
\hline
\end{tabular}

heterogeneously methylated epialleles, DNAs from normal prostate and malignant cancer cell lines were investigated. In LNCaP cells FAM-signals with respective high signal strength were mainly observed using probe PLA2R1-3 (Figure 5, left panel labeled with PLA2R1-3). Probes PLA2R1-1b/2b/2c generated low levels of FAM-signals and probes PLA2R1-1a/1c/0 gave no signal (Figure 5). In PrEC low levels of PLA2R1-3-specific signals, but significant levels of PLA2R1-1c- and PLA2R1-1a-specific signals were visible (Figure 5). In comparison the level of PLA2R1-3epiallele was higher in BPH-1 (Figure 5), but significantly lower to those present in the malignant LNCaP, PC-3 and DU-145 cells containing high levels of fully methylated PLA2R1-3 epialleles (Figure 5).

Probe PLA2R1-2c produced FAM signals with high strength (above the thresholds) specific for the PLA2R1-2c epiallele (labeled with 2c-I in Figure 5); two additional subfractions below the thresholds were also present (Figure 5, right panel PLA2R1-2c labeled with 2c-II and 2c-III). The level of signals of these subfractions was consistent with those found in the blood sample E188 using the same probe (Figure 4, left panel labeled with PLA2R1-2C). The subfraction with FAM signal strength moderately above the strength of empty droplets (labeled with 2c-III in Figure 5) can be assigned to the PLA2R1-3 fraction based on the comparison with the amount of FAM signals of this fraction present in LNCaP cells (Figure 5).

Furthermore, three FAM-positive subfractions resulting from the use of the PLA2R1-3 probe were defined (labeled with 3-I, 3-II, and 3-III in Figure 5). On the basis that the PLA2R1-3 probe generates FAM signals with low amplitude (subfractions 3-II and 3-III in Figure 5) through mismatching with PLA2R 1-2a/2b/2c epialleles and in PC-3 cells only the subfraction 3-II was present with amount of FAM signals 
PLA2R1-1a

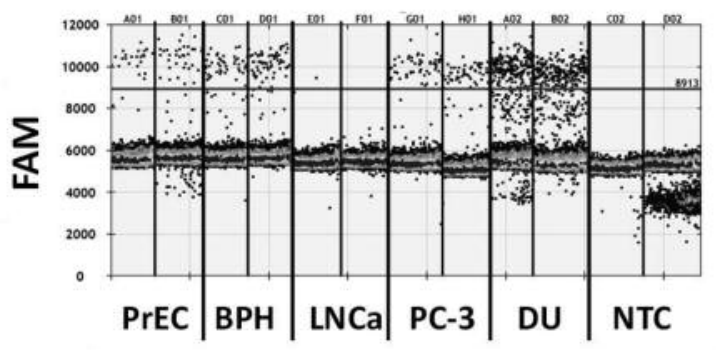

PLA2R1-1C

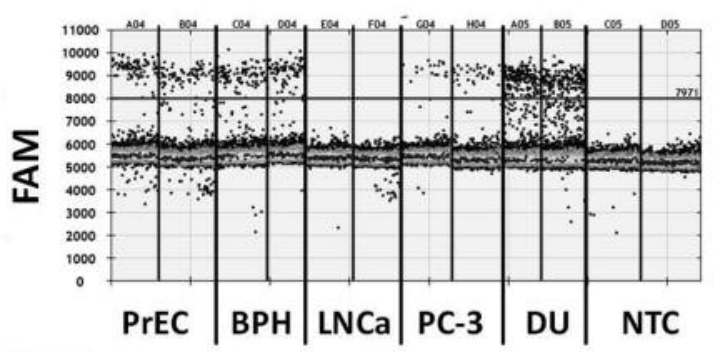

PLA2R1-2b

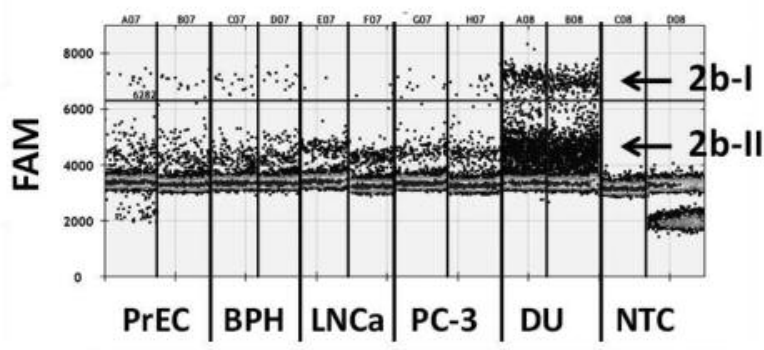

PLA2R1-3

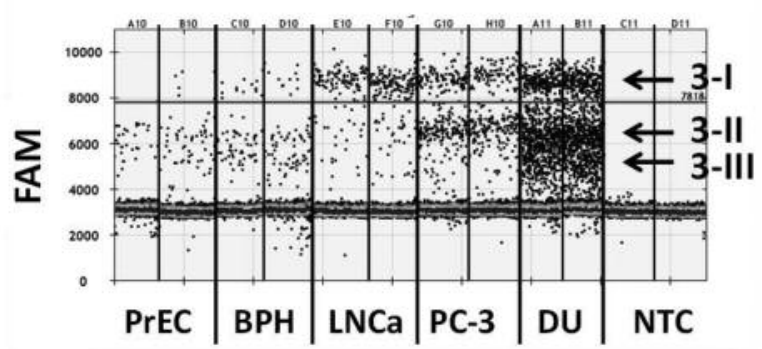

PLA2R1-1b

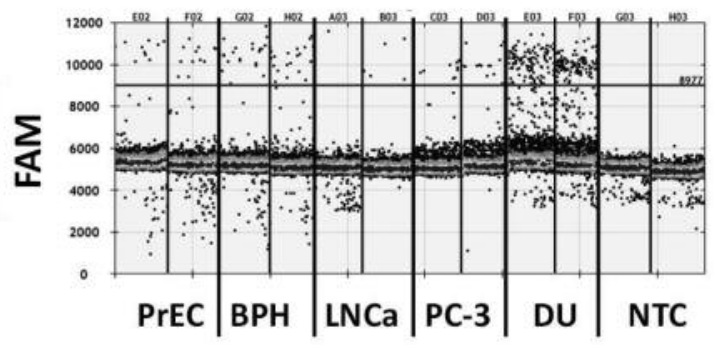

PLA2R1-2a

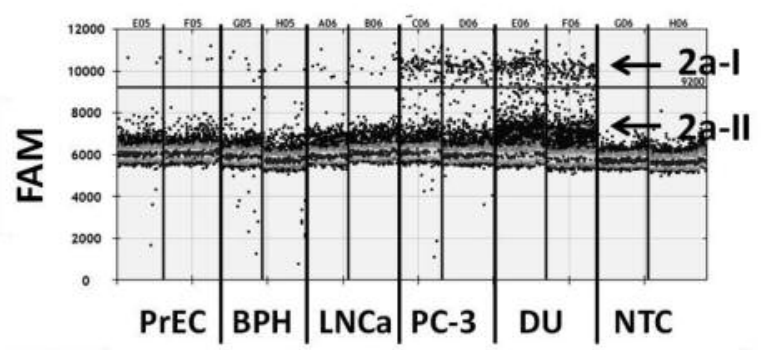

PLA2R1-2c

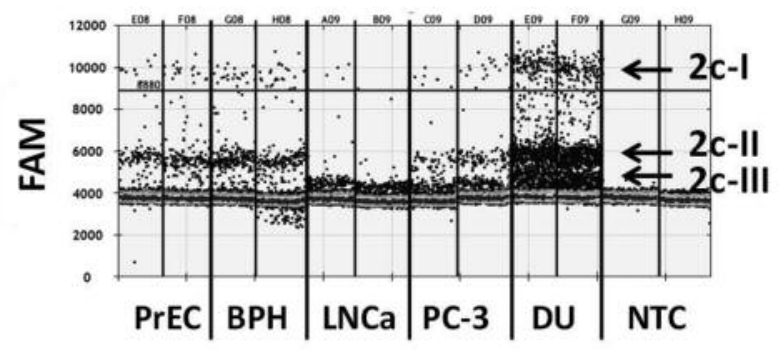

PLA2R1-0

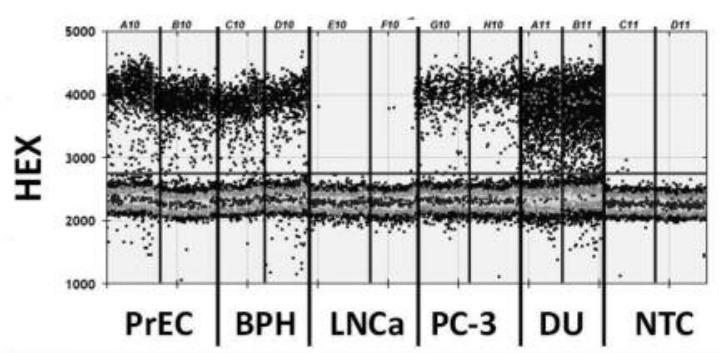

Figure 5. Quantification of FAM-signals (positive for heterogeneously methylated epialleles) and HEX-signals (positive for the unmethylated epiallele) in DNA samples isolated from normal epithelial cells of prostate (PrEC), benign prostate hyperplasic cell line BPH-1 (BPH), and malignant LNCaP (LNCa), PC-3 (PC-3) and DU-145 (DU) prostate cell lines after 40 cycles of ddPCR. Applied thresholds are shown in black. Arrows labeled with PLA2R1-2a, PLA2R1-2b, PLA2R1-2c, and PLA2R1-3 indicate subfractions with high (above threshold) and low FAM signal amplitudes (below threshold) generated by mismatching of probes with epialleles. DNA from prostate cell lines was analyzed in duplicate and results are representative of two independent experiments. NTC, Non-template controls. 
A

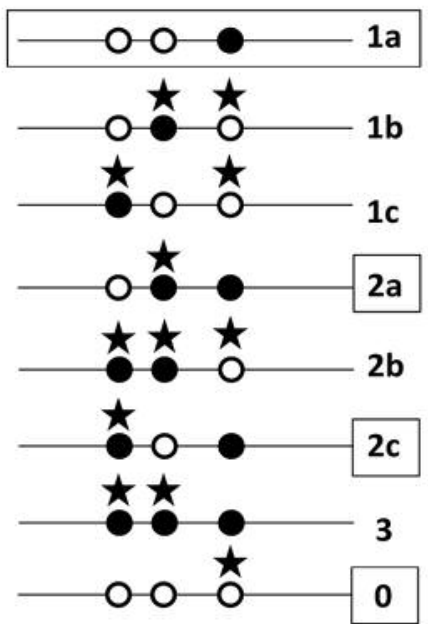

D

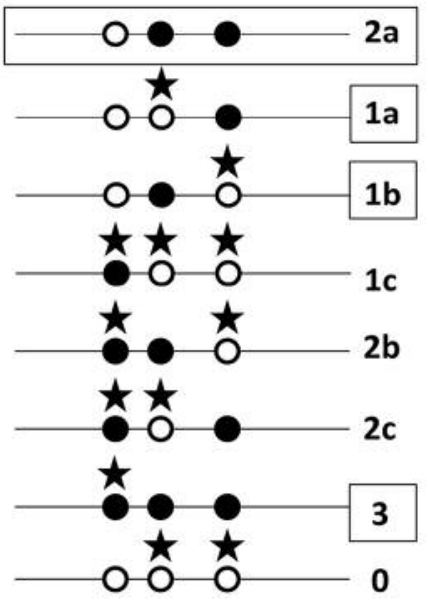

B

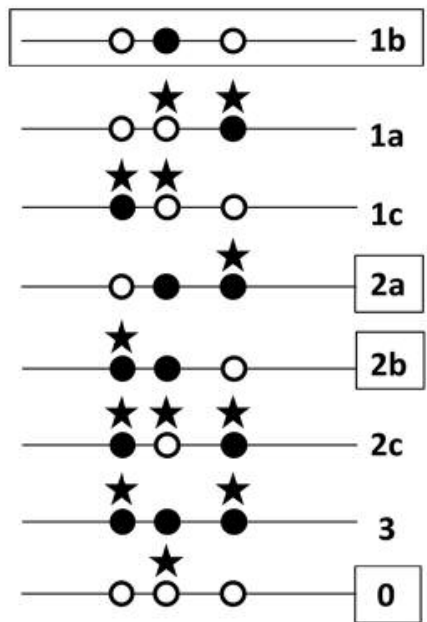

E

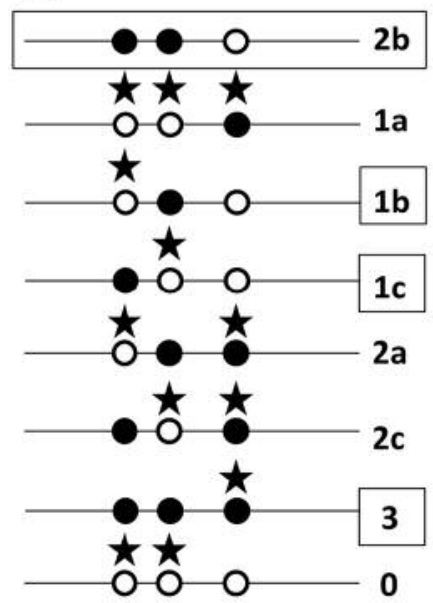

C

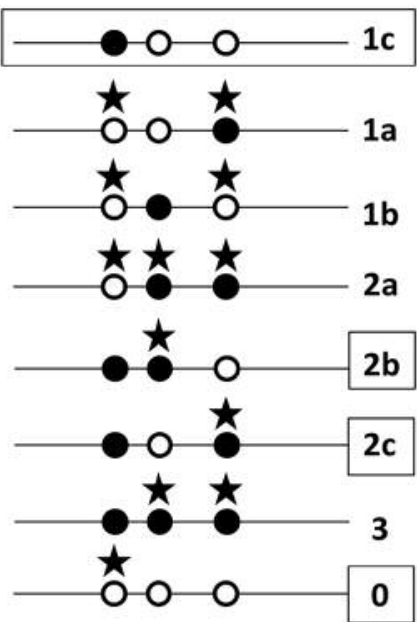

$\mathbf{F}$

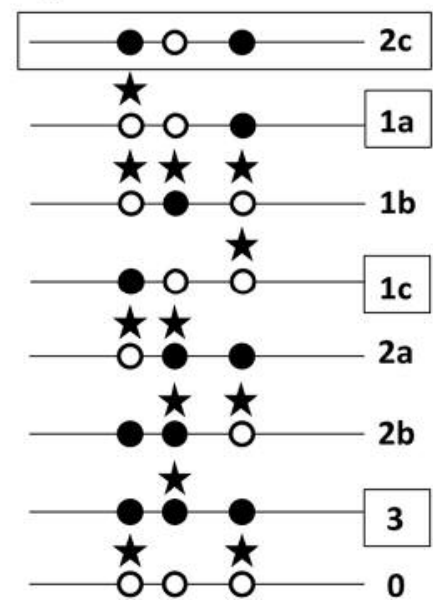

G

H
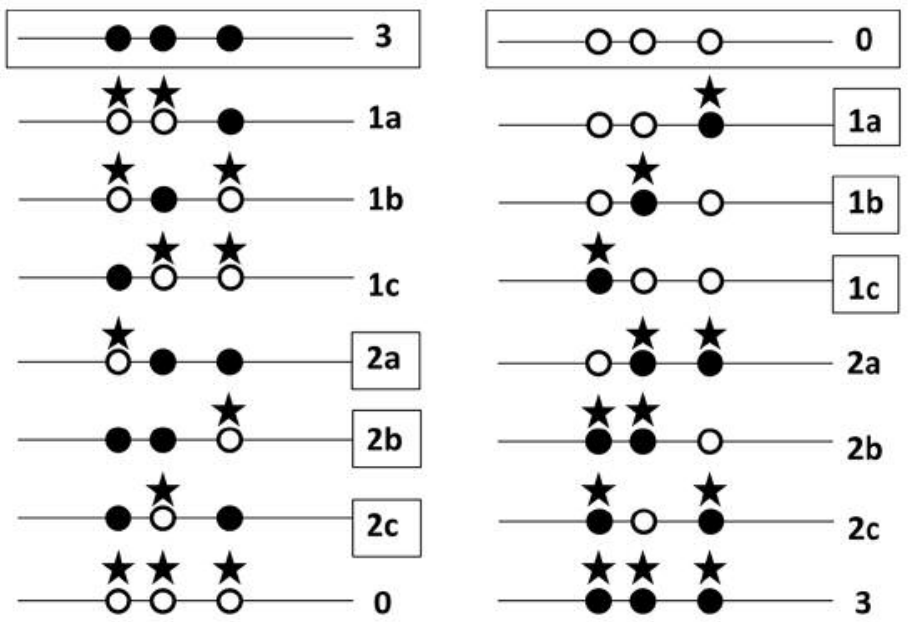

Figure 6. Possible constellations giving low amplitude FAM-positive signals due to mismatching caused by a single nucleotide difference between the eight applied probes and the methylated epialleles. (A-H) show the eight probes with the top row indicating the intended matching; below positions of mismatching are marked with stars. On the right side, epialleles characterized by a single nucleotide difference to the respective probe are framed. 
Table III. Percentage amounts shown as mean \pm SD of epiallele copies in DNA samples isolated from PrEC - normal epithelial cells of the prostate, BPH-1 - benign hyperplasia of the prostate $(C)$, and malignant prostate cancer cell lines LNCaP, PC-3, and DU-145. Following bisulfite modification genomic DNA samples were analyzed using ddPCR with eight different fluorescence-labelled probes (refer to Table I). Analyses were performed in duplicate and results are representative of two independent experiments.

\begin{tabular}{lrrlrr}
\hline Probe ID & PrEC & \multicolumn{1}{c}{ BPH-1 } & LNCaP & PC-3 & DU-145 \\
\hline PLA2R1-1a & $3.6 \pm 1.1$ & $8.0 \pm 1.0$ & $0.8 \pm 1.1$ & $8.6 \pm 0.8$ & $7.4 \pm 0.5$ \\
PLA2R1-1b & $1.3 \pm 0.2$ & $2.6 \pm 0.2$ & $2.1 \pm 1.9$ & $2.1 \pm 0.2$ & $3.7 \pm 0.1$ \\
PLA2R1-1c & $8.9 \pm 0.4$ & $12.2 \pm 1.1$ & 0 & $4.3 \pm 1.6$ & $6.7 \pm 0.4$ \\
PLA2R1-2a & $0.5 \pm 0.2$ & $1.0 \pm 0.2$ & $8.8 \pm 2.3$ & $15.5 \pm 1.7$ & $4.0 \pm 0.8$ \\
PLA2R1-2b & $1.3 \pm 0.4$ & $1.7 \pm 0.3$ & $2.2 \pm 1.1$ & $2.3 \pm 1.0$ & $6.3 \pm 0.6$ \\
PLA2R1-2c & $2.0 \pm 0.8$ & $2.6 \pm 0.1$ & $2.0 \pm 1.1$ & $1.7 \pm 0.7$ & $4.1 \pm 0.3$ \\
PLA2R1-3 & $0.4 \pm 0.2$ & $1.5 \pm 0.2$ & $82.9 \pm 29.3$ & $15.3 \pm 1.8$ & $10.3 \pm 1.2$ \\
PLA2R1-0 & $82.0 \pm 7.0$ & $70.4 \pm 8.0$ & $1.3 \pm 1.1$ & $50.2 \pm 4.1$ & $57.4 \pm 4.1$ \\
\hline
\end{tabular}

which matches with those of the PLA2R1-2a epiallele in these cells this cluster below threshold can be assigned to the PLA2R1-2a epiallele.

In addition to this subfraction a further distinct subfraction (labelled with 3-III in Figure 5) was present in DU-145 cells. This additional subfraction did not occur in PC-3 cells. Because in the latter the concentration of PLA2R1-2b and PLA2R1-2c epialleles was low, this subfraction, which is seen separately in the DU-145 cells, should be the PLA2R1$2 \mathrm{~b}$ or PLA2R1-2c epiallele. This conclusion is consistent with signal levels observed using PLA2R1-2b and PLA2R12c probes in this cell line (Figure 5).

Quantification of individual epialleles in DNA from peripheral blood, bone marrow aspirate, normal and malignant prostate cancer cell lines. The percentages of the individual epialleles in DNA of blood cells, bone marrow and prostate cells that were measured using the new ddPCR are summarized in Tables II and III. The data showed that with the exception of PLA2R1-2a epiallele the percentage of all heterogenously methylated epialleles (PLA2R1-1a, PLA2R1-1b, PLA2R1-1b, PLA2R1-2b and PLA2R1-2c) was higher in blood leukocytes in comparison to bone marrow DNA. Simultaneously, the percentage of fully methylated PLA2R1-3 epiallele in blood leukocyte DNA was significantly lower (Table II).

In PrEC and BPH-1 cells the level of PLA2R1-1a/1b/1c epialleles in relation to PLA2R1-2a/2b/2c and PLA2R1-3 was higher, whereas in LNCaP, PC-3 and DU-145 malignant prostate cell lines the level of epialleles with two and three methylated CpG sites (PLA2R1-2a/2b/2c and PLA2R1-3) was significantly higher in comparison to epiallels with one methylated $\mathrm{CpG}$ site (PLA2R1-1a/1b/1c), respectively (Table III).
Table IV. Recoveries (\%) after summation of copy numbers of completely methylated epiallele PLA2R1-3 (3) and PLA2R1-2a/2b/2c epialleles $(2 a+2 b+2 c)$ alone and together with PLA2R1-1a/1b/1c epialleles $(1 a+1 b+1 c)$ presented as mean \pm S.E. Copy amounts of epialleles measured separately with probes PLA2RI-3 (using the threshold as shown in Figure 4 for PLA2R1-3s and Figure 5 for PLA2R1-3), PLA2R1-2a/2b/2c, and PLA2R1-1a/1b/1c were related to copy numbers quantified using all PLA2R1-3-dependent FAM-positive signals (using threshold as shown in Figure 4 for PLA2R1-3w and Figure 5 for PLA2R1-3 including subfractions 3-II and 3-III); n.d. - not determine due to low amounts of epialleles PLA2R1-2a/2b/2c and PLA2R1-1a/1b/1c in LNCaP cells.

\begin{tabular}{lcc}
\hline Samples & $\begin{array}{c}\text { Epialleles } \\
3+(2 a+2 b+2 c)\end{array}$ & $\begin{array}{c}\text { Epialleles } \\
\end{array}$ \\
\hline E188 & $93 \pm(2 a+2 b+2 c)+(1 a+1 b+1 c)$ \\
K189 & $93 \pm 4$ & $138 \pm 4$ \\
PrEC & $103 \pm 6$ & $93 \pm 5$ \\
BPH-1 & $98 \pm 8$ & $450 \pm 9$ \\
LNCaP & n.d. & $424 \pm 2$ \\
PC-3 & $96 \pm 20$ & n.d. \\
DU-145 & $104 \pm 6$ & $136 \pm 15$ \\
\end{tabular}

To figure out the origin of the two additional FAM-positive subfractions with lower amplitude (two arrows in Figure 4, left panel labeled with PLA2R1-3w, three arrows labeled with 3-I-III in Figure 5, left panel with PLA2R1-3) using PLA2R1-3 probe amounts of the PLA2R1-2a/2b/2c epialleles alone and together with those of PLA2R $1-1 \mathrm{a} / 1 \mathrm{~b} / 1 \mathrm{c}$ epialleles were added to copy amounts of the PLA2R1-3 epiallele (Table IV). The resulting amounts were related to copy amounts calculated using thresholds that included all FAM positive signals in the calculation (Figure 4 marked with PLA2R1-3s and PLA2R1-3w). The recovery averaged $99.0 \%$ for sample ID E188, if the amounts of PLA2R1-2a/2b/2c epialleles were added to those of the PLA2R1-3 epiallele (Table IV). If copies of the PLA2R1-1a/1b/1c epialleles were included in the calculations, the recovery rate increased to $141 \%$ for this sample. In case of bone marrow sample ID K189 the proportion of these epialleles was too low, to may have a significant effect on the recovery rate. In this sample, DNA was nearly completely methylated with $98.2 \%$ which was consistent to MS-HRM analysis (Figure 2B).

A similar recovery as in sample ID E188 was received in DNA samples of PrEC (103\%), BPH-1 (98\%), PC-3 (96\%) and DU-145 (103\%) cells (Table IV). By consideration of PLA2R1-1a/1b/1c-epiallel copies into the calculation recovery rates of $450 \%, 424 \%, 136 \%$ and $173 \%$ in cases of PrEC, BPH-1, PC-3 and DU-145 cells resulted, respectively (Table IV). Comparable to the bone marrow sample ID $\mathrm{K} 189$ the amount of PLA2R1-1a/1b/1c epialleles was with $2.9 \%$ to low in LNCaP cells to have an effect on the recovery (Figure 5). 
As data demonstrated that FAM signals are generated in addition to complementary through mismatching based on a single nucleotide difference, possible constellations using the eight probes are summarized in Figure 6.

\section{Discussion}

The presented study describes a new technique for identification and quantification of heterogeneously methylated epialleles based on digital PCR with fluorescencelabeled probes complementary to the sequences of the epialleles. Heterogeneously methylated epialleles can occur when at least two CpGs are evaluated within a sequence. Recently, a strong different methylation of two CpG sites in the SERPINA5 promoter connected with the presence of heterogeneously methylated epialleles was shown in the aggressive prostate cancer cell lines DU-145 and PC-3 in comparison to normal epithelial and benign hyperplastic prostate cells (22). For quantification of epialleles $2^{\mathrm{n}}$ probes, with $\mathrm{n}$ being the number of $\mathrm{CpGs}$ are required.

In the present study, we have assessed three $\mathrm{CpG}$-sites belonging to a previously investigated cluster of four $\mathrm{CpG}$ sites in the promoter of PLA2R1 (19). The cluster is part of a bigger $\mathrm{CpG}$ island and was found to be methylated more often in leukemia than other $\mathrm{CpGs}$ within the island. For three $\mathrm{CpG}$ sites we have generated eight probes, one for the unmethylated allele and seven complementary to the heterogeneously methylated epialleles (Figure 1 and Table I). Analysis of the $P L A 2 R 1$ promoter methylation in blood and bone marrow DNA of a patient with B-ALL showed that bone marrow DNA was predominantly methylated in the investigated region, whereas blood leukocyte DNA presents with a complex MSHRM profile suggesting heterogeneously methylated epialleles (Figure 2). MS-HRM was, however, not able to quantify these epialleles. In order to overcome this limitation, we have developed a ddPCR method utilizing FAM and HEX labeled probes for homogenously methylated/unmethylated and heterogeneously methylated alleles.

DNA samples from bone marrow cells and blood leukocytes of the B-ALL patient were utilized to determine the specificity of the new method in a proof of concept. We showed that besides the PLA2R1-3 probe the dominant subfraction of completely methylated epialleles in bone marrow DNA was mismatched by PLA2R $1-2 a / 2 b / 2 c$ probes. This mismatching was associated with significantly lower signal amplitude and therefore allowed for the discrimination between specific and unspecific fluorescence signals. In addition, no FAM-positive cluster, which could have been assigned to the dominant fraction of completely methylated epiallele in bone marrow DNA, was generated by PLA2R11a/1b/1c probes. Similar results were observed in LNCaP cells suggesting that FAM signals with low amplitude were generated by mismatching on the basis of a single but not on dual nucleotide sequence differences between probes and analyzed epialleles under the used ddPCR conditions. This agrees with estimation of the recovery by addition of the copy amounts of epialleles with two methylated $\mathrm{CpG}$ sites and copies of fully methylated epialleles resulting in a recovery of nearly $100 \%$ in relation to the calculated copy amounts by including all positive FAM-signals. To prove the specificity of the used probes in more detail, however, studies using control DNA for the individual epialleles generated by oligonucleotide directed mutagenesis are necessary in the future.

In comparison to bone marrow cells containing mainly completely methylated epialleles, DNA from blood leukocytes of the same patient was characterized by remarkable elevated levels of heterogeneously methylated epialleles. Although MS-HRM could detect heterogeneously methylated epialleles, our new method was able to quantify the different epialleles. Mechanisms explaining the elevated levels of heterogeneously methylated epialleles in peripheral blood (containing $4 \%$ blasts) in comparison to bone marrow (with $61 \%$ blasts) are still unresolved. One explanation might be that bone marrow derived blood cells lose some of their methylation during maturation increasing the levels of heterogeneously methylated epialleles at expense of fully methylated DNA. Similarly, the question of the pathophysiological relevance of heterogeneously methylated epialleles is still largely unanswered (9).

Comparing MS-HRM and ddPCR-based data it becomes obvious that three CpG sites analyzed in the ddPCR (\#4-\#6 according to our nomenclature in Figure 1) were predominantly methylated in the studied malignant prostate cell lines, whereas the adjacent CpG sites (\#1-\#3 and \#7-\#9 in Figure 1) in PC-3 and DU-145 cells and also in part in LNCaP cells were not fully methylated. This explains the near but not full overlap of their melt curves with the $100 \%$ methylated control DNA in the MS-HRM analysis (Figure 2B).

The proposed new technique which we named epiallelesensitive droplet digital PCR (EAST-ddPCR) is a closed-tube technique minimizing potential contamination between patient samples. In addition, this technique overcomes the problem of PCR bias associated with conventional PCRbased techniques, as there is no competition between DNA templates and primer pair (23-32). In comparison to digital MS-HRM, which allows analysis only in limited compartments like in 96-reaction-well-plates, EAST-ddPCR can be performed in 20,000 up to several $10^{6}$ compartments resulting in significantly higher analytical sensitivities and reproducibility. Sensitivity was determined below $1 \%$, whereas other techniques like Next Generation Sequencing, Pyrosequencing, and SequenomMassArray exhibit sensitivities of $5-10 \%$, also connected to high initial investment and consumable costs for the analysis of heterogeneously methylated epialleles $(9,12-14)$. 
A further alternative approach called EpiQwas previously described based on ligation of complementary and fluorescence-labeled probes by DNA ligase technique also allowing quantification of epialleles without sequencing or following HRM analysis $(33,34)$. The assay exhibited a high specificity, but the authors stated that when one target was in excess the assay did not accurately measure the amount of each target in the sample. The analytical sensitivity was given as $5-10 \%$ of differences in epiallele levels. An additional problem is the two step nature of the assay with conventional PCR at the beginning resulting in a possible bias towards certain epialleles (23-31). PCR may amplify targets with varying efficiency creating a bias before the analytical step of DNA ligase. In our presented method quantification of epialleles is occurring directly during ddPCR by hybridization of probes thereby preserving the advantage of PCR amplification without potential bias.

In conclusion, ddPCR using a set of fluorescencelabeled probes can be used for quantification of heterogeneously methylated epialleles with acceptable sensitivities and specificities. If multicolor flow cytometry (ideally with eight channels) is used, a master mix with simultaneously all fluorescence-labeled probes can be applied resulting in acceptable costs for these analyses. Small changes in the pattern of heterogeneously methylated epialleles are detectable. The principle of EAST-ddPCR can be transferred to other genes if the sequence of the target gene contains at least two $\mathrm{CpG}$ sites of interest in a region of 30 nucleotides which is the preferred length of probes.

Our newly developed EAST-ddPCR provides a platform for studying the generation and regulation of heterogeneously methylated epialleles during developmental and pathological processes. It has the potential to improve our currently incomplete understanding of such epialleles in carcinogenesis. In addition to monitoring the pattern of epialleles for therapy control, for example of azacitidine-treated leukemia patients, the quantification of heterogeneously methylated epialleles may be possibly helpful for the identification of new biomarkers specific for particular epialleles improving the early diagnosis of benign and malignant diseases such as benign prostate hyperplasia and prostate cancer.

\section{Funding}

This work was supported by Grant from Bundesministerium für Bildung und Forschung (BMBF) and Forschungszentrum Jülich GmbH (031B0098C, Germany) (to M.M.).

\section{Conflicts of Interest}

The Authors declare that they have no conflicts of interest.

\section{Acknowledgements}

The Authors are grateful to Romy Adler for her expert technical assistance.

\section{References}

1 Laird PW: The power and the promise of DNA methylation markers. Nat Rev Cancer 3: 253-266, 2003.

2 Shames DS, Minna JD and Gazdar AF: DNA methylation in health, disease, and cancer. Curr Mol Med 7: 85-102, 2007.

3 Tost J: DNA methylation: an introduction to the biology and the disease-associated changes of a promising biomarker. Mol Biotechnol 44: 71-81, 2010.

4 Kim H, Wang X and Jin P: Developing DNA methylation-based diagnostic biomarkers. J Genet Genomics 45: 87-97, 2018.

5 Widschwendter M, Jones A, Evans I, Reisel D, Dillner J, Sundström K, Steyerberg EW, Vergouwe Y, Wegwarth O, Rebitschek FG, Siebert U, Sroczynski G, de Beaufort ID, Bolt I, Cibula D, Zikan M, Bjørge L, Colombo N, Harbeck N, Dudbridge F, Tasse AM, Knoppers BM, Joly Y, Teschendorff AE, Pashayan $\mathrm{N}$ and FORECEE (4C) Consortium: Epigenome-based cancer risk prediction: rationale, opportunities and challenges. Nat Rev Clin Oncol, 2018. https://doi.org/ 10.1038/nrclinonc.2018.30

6 Assenov Y, Brocks D and Gerhäuser C: Intratumor heterogeneity in epigenetic patterns. Semin Cancer Biol, 2018. https:// doi.org/10.1016/j.semcancer.2018.01.010

7 Sukocheva O, Menschikowski M, Hagelgans A, Yarla NS, Siegert G, Reddanna P and Bishayee A: Current insights into functions of phospholipase A2 receptor in normal and cancer cells: More questions than answers. Semin Cancer Biol, 2018. https:// doi.org/10.1016/j.semcancer. 2017.11.002

8 Lapinska K, Faria G, McGonagle S, Macumber KM, Heerboth S and Sarkar S: Cancer Progenitor Cells: The Result of an Epigenetic Event? Anticancer Res 38: 1-6, 2018.

9 Mikeska T, Candiloro IL and Dobrovic A: The implications of heterogeneous DNA methylation for the accurate quantification of methylation. Epigenomics 2: 561-573, 2010.

10 Candiloro IL, Mikeska T, Hokland P and Dobrovic A: Rapid analysis of heterogeneously methylated DNA using digital methylation-sensitive high resolution melting: application to the CDKN2B (p15) gene. Epigenetics Chromatin 1: 7, 2008.

11 Weisenberger DJ, Trinh BN, Campan M, Sharma S, Long TI, Ananthnarayan D, Liang G, Esteva FJ, Hortobagyi GN, McCormick F, Jones PA and Laird PW: DNA methylation analysis by digital bisulfite genomic sequencing and digital MethyLight. Nucleic Acids Res 36: 4689-4698, 2008.

12 Fleitas T, Ibarolla-Villava M, Ribas $G$ and Cervantes A: MassARRAY determination of somatic oncogenic mutations in solid tumors: Moving forward to personalized medicine. Cancer Treat Rev 49: 57-64, 2016.

13 Wang L, Lin S, Zhang J, Tian T, Jin L and Ren A: Fetal DNA hypermethylation in tight junction pathway is associated with neural tube defects: A genome-wide DNA methylation analysis. Epigenetics 12: 157-165, 2017.

14 Ehrich M, Nelson MR, Stanssens P, Zabeau M, Liloglou T, Xinarianos G, Cantor CR, Field JK and van den Boom D: Quantitative high-throughput analysis of DNA methylation patterns by base-specific cleavage and mass spectrometry. Proc Natl Acad Sci USA 102: 15785-15790, 2005. 
15 Tamaru S, Mishina H, Watanabe Y, Watanabe K, Fujioka D, Takahashi S, Suzuki K, Nakamura T, Obata JE, Kawabata K, Yokota Y, Murakami M, Hanasaki $\mathrm{K}$ and Kugiyama K: Deficiency of phospholipase A2 receptor exacerbates ovalbumininduced lung inflammation. J Immunol 191: 1021-1028, 2013.

16 Bernard D and Vindrieux D: PLA2R1: Expression and function in cancer. Biochim Biophys Acta 1846: 40-44, 2014.

17 Augert A, Payré C, de Launoit Y, Gil J, Lambeau G and Bernard D: The M-type receptor PLA2R1 regulates senescence through the p53 pathway. EMBO Rep 10: 271-277, 2009.

18 Vindrieux D, Augert A, Girard CA, Gitenay D, Lallet-Daher H, Wiel C, Le Calvé B, Gras B, Ferrand M, Verbeke S, de Launoit Y, Leroy X, Puisieux A, Aubert S, Perrais M, Gelb M, Simonnet H, Lambeau $\mathrm{G}$ and Bernard D: PLA2R1 mediates tumor suppression by activating JAK2. Cancer Res 73: 6334-6345, 2013.

19 Menschikowski M, Platzbecker U, Hagelgans A, Vogel M, Thiede C, Schönefeldt C, Lehnert R, Eisenhofer G and Siegert G: Aberrant methylation of the M-type phospholipase A(2) receptor gene in leukemic cells. BMC Cancer 12: 576, 2012.

20 Menschikowski M, Hagelgans A, Nacke B, Jandeck C, Sukocheva O and Siegert G: Epigenetic control of phospholipase A2 receptor expression in mammary cancer cells. BMC Cancer 15: 971, 2015.

21 Vindrieux D, Devailly G, Augert A, Le Calvé B, Ferrand M, Pigny P, Payen L, Lambau G, Perrais M, Aubert S, Simonnet H, Dante R and Bernard D: Repression of PLA2R1 by c-MYC and HIF-2 $\alpha$ promotes cancer growth. Oncotarget 5: 1004-1013, 2014.

22 Hagelgans A, Jandeck C, Friedemann M, Donchin A, Richter S and Menschikowski $\mathrm{M}$ : Identification of $\mathrm{CpG}$ Sites of SERPINA5 Promoter with Opposite Methylation Patterns in Benign and Malignant Prostate Cells. Anticancer Res 37: 66096618, 2017.

23 Kraytsberg Y and Khrapko K: Single-molecule PCR: an artifactfree PCR approach for the analysis of somatic mutations. Expert Rev Mol Diagn 5: 809-815, 2005.

24 Warnecke PM, Stirzaker C, Melki JR, Millar DS, Paul CL and Clark SJ: Detection and measurement of PCR bias in quantitative methylation analysis of bisulphite-treated DNA. Nucleic Acids Res 25: 4422-4426, 1997.
25 Warnecke PM, Stirzaker C, Song J, Grunau C, Melki JR and Clark SJ: Identification and resolution of artifacts in bisulfite sequencing. Methods 27: 101-107, 2002.

26 Shen L, Guo Y, Chen X, Ahmed S and Issa JP: Optimizing annealing temperature overcomes bias in bisulfite PCR methylation analysis. Biotechniques 42: 48, 50, 52 passim, 2007.

27 Wojdacz TK and Dobrovic A: Methylation-sensitive high resolution melting (MS-HRM): a new approach for sensitive and high-throughput assessment of methylation. Nucleic Acids Res 35: e41, 2007.

28 Wojdacz TK, Hansen LL and Dobrovic A: A new approach to primer design for the control of PCR bias in methylation studies. BMC Res Notes 1: 54, 2008.

29 Wojdacz TK, Dobrovic A and Hansen LL: Methylation-sensitive high-resolution melting. Nat Protoc 3: 1903-1908, 2008.

30 Wojdacz TK and Hansen LL: Reversal of PCR bias for improved sensitivity of the DNA methylation melting curve assay. Biotechniques 41: 274-278, 2006.

31 Wojdacz TK, Borgbo T and Hansen LL: Primer design versus PCR bias in methylation independent PCR amplifications. Epigenetics 4: 231-234, 2009.

32 Candiloro ILM, Mikeska $\mathrm{T}$ and Dobrovic A: Assessing alternative base substitutions at primer $\mathrm{CpG}$ sites to optimise unbiased PCR amplification of methylated sequences. Clin Epigenetics 9: 31, 2017.

33 Palanisamy R, Connoly AR and Trau M: Epiallele quantification using molecular inversion probes. Anal Chem 83: 2631-2637, 2011.

34 Wee EJ, Rauf S, Shiddiky MJ, Dobrovic A and Traut M: DNA ligase-based strategy for quantifying heterogeneous DNA methylation without sequencing. Clin Chem 61: 163-171, 2015.

Received February 1, 2018

Revised March 28, 2018

Accepted April 23, 2018 